\title{
Testicular Regression
}

National Cancer Institute

\section{Source}

National Cancer Institute. Testicular Regression. NCI Thesaurus. Code C120200.

A condition characterized by typical male genital appearance in a $46, X Y$ individual in whom testes are unable to be located. 